\title{
Escalas de desarrollo y construcción de una herramienta tecnológica dirigida a la estimulación de la niñez con riesgo biológico neonatal
}

Ana Lupita Garrido Sandino', Linda María Madriz Bermúdez²

1. Escuela La Trinidad, Ministerio de Educación Pública (MEP)

Escuela de Ciencias de la Educación (ECE), Universidad Estatal a Distancia (UNED), algarrido@hotmail.com 2. Cátedra de Conceptualización de la Educación Especial, Universidad Estatal a Distancia (UNED), Imadriz@uned.acr

\section{Resumen}

Existen numerosas investigaciones y estudios que hacen referencia a la plasticidad cerebral como forma de favorecer la habilitación y rehabilitación de estructuras y funciones que se pueden ver comprometidas ante la presencia de factores de riesgo biológico neonatal. La detección oportuna de éstos permite la estimulación y atención temprana de las áreas que se ven afectadas de modo que se minimice la presencia de secuelas temporales o permanentes que puedan derivar en una condición de discapacidad.

La investigación desarrollada por Baxter, Madriz, Meléndez, Mora, Hidalgo, y Solano (2013) muestra la importancia de la atención temprana dirigida a esta población infantil así como la necesidad de que las familias se involucren. Por tanto, se requiere de la valoración del desarrollo infantil por medio de escalas válidas y confiables. Se realiza una revisión sistemática de doce investigaciones referentes a la aplicación de escalas de desarrollo infantil y la estimulación temprana, publicadas en Latinoamérica y España. Asimismo, se presenta la propuesta de los investigadores costarricenses para desarrollar una herramienta web de valoración y estimulación temprana.

Palabras clave: Estimulación y atención Temprana, Condición de riesgo biológico, Discapacidad, Prevención, Escala de desarrollo infantil.

\section{Abstract \\ Scales development and construction of a technological tool aimed at stimulating children with neonatal biohazard}

Many studies refer to brain plasticity which enables the structures and functions that might be compromised due to neonatal biological risk conditions. The appropriate detection of these conditions allows stimulation and early attention to act on jeopardize areas, in order to minimize temporal or permanent aftermaths presence that could develop into disability. Baxter, Madriz, Melendez, Mora, Hidalgo, \& Solano (2013) investigation shows the importance of the early attention on the child population and the necessity for families to involve in the process too. Therefore several valid and reliable scales are required to test the child development. For the purpose of this article, twelve investigations that focus on applied child development scales and early stimulation published in Latin America and Spain were consulted. In addition, this article also describes the work done by the Costa Rican investigators in order to develop a web tool for assessment and early stimulation.

Key words: Early Stimulation, Biohazard condition, Disability, Prevention, Child development scale. 


\section{INTRODUCCIÓN}

El presente trabajo surge a partir de la investigación "Estudio comparativo acerca de la implementación de programas de estimulación temprana para niños prematuros atendidos en el Estancia Infantil № 2 de la ciudad de Puebla (México) y el CINAI de la ciudad de Goicoechea (Costa Rica): una propuesta de atención temprana para la prevención de la discapacidad" desarrollada entre los años 2011 y 2013. Los resultados obtenidos señalan la necesidad de iniciar una segunda fase de la investigación que continúe al proceso iniciado, en la que se elabore una herramienta tecnológica para la valoración, estimulación y atención temprana de la niñez en general, pero en particular para aquella que presenta una condición de riesgo biológico en su nacimiento de modo que se prevenga la aparición de una condición de discapacidad posterior. El término riesgo biológico, tal y como se explica más adelante, hace referencia a la presencia de una o más características o factores que incrementan la posibilidad de que una persona o un grupo puedan tener un daño en su salud. En este caso, se alude a las condiciones que se presenten en los períodos pre, peri o postnatal tales como: prematuridad, bajo peso al nacer, hipoxia o anoxia.

Los avances en la medicina y la tecnología han propiciado una disminución en la tasa de la mortalidad neonatal, logrando la supervivencia de la niñez expuesta a riesgos biológicos. Sin embargo, conlleva nuevos retos ante la presencia de secuelas relacionadas con el desarrollo, las cuales eventualmente podrían derivar en una condición de discapacidad.

La detección precoz de posibles alteraciones en el desarrollo infantil permite la intervención oportuna en una etapa en la que el cerebro se encuentra en un período crítico de crecimiento y de plasticidad. Toma importancia la atención temprana, que autores como Katz (2005), Poblano (2003) y Zuluaga (2001) consideran contribuye a reducir los efectos de una lesión, disminuyendo posibles secuelas permanentes. De igual manera, tanto las familias como las educadores y médicos participantes en la investigación desarrollada por Baxter et al. (2013) coinciden en esta afirmación al considerar que puede evitar alguna condición de discapacidad.

De igual forma, otro resultado de la citada investigación alude a la percepción de los participantes de que se requiere una coordinación entre los diferentes servicios médicos y educativos involucrados en la atención de la primera infancia que presenta una condición de riesgo biológico neonatal. Sin embargo, se valora que la ejecución de los programas de estimulación temprana en ambos países ha sido adecuada, aunque se señala que a las familias se les brinda poca información sobre las implicaciones de un nacimiento con factores de riesgo biológico asociado, la necesidad de la estimulación temprana y la forma que ellos pueden fortalecer el desarrollo infantil con prácticas afines a la estimulación y atención temprana. (Baxter et al., 2013).

En el marco de estos resultados es que los investigadores se plantean la necesidad de construir e implementar una herramienta tecnológica de valoración, estimulación y atención temprana dirigida a las familias y cuidadores de la niñez que presenta una condición de riesgo biológico neonatal que, de manera colateral se constituya en un proceso de empoderamiento parental y de capacitación familiar.

El diseño de la herramienta web va a permitir que los interesados accedan a la información en el momento que lo requieran, sin importar la ubicación geográfica o el nivel socioeconómico, con lo que se aseguraría su alcance universal mediante el dominio web y el soporte tecnológico de la Universidad Estatal a Distancia (UNED), Costa Rica. Se propone un acceso asincrónico mediante plataforma móvil y fija. El uso del instrumento es extensivo a profesionales en las áreas de salud y educación pues contempla la posibilidad de valorar el nivel de desarrollo de la primera infancia en las diferentes áreas, de modo que se puedan estimular las que se encuentren funcionando por debajo de lo esperado para la edad cronológica.

Además, el proyecto se circunscribe en una alianza estratégica que incluye a representantes de Caja Costarricense del Seguro Social (CCSS) y la Fundación Hera, entidades que permiten ampliar el rango de cobertura de la población y particularmente el seguimiento y la atención integral de los usuarios de la herramienta. En el caso de la CCSS, por medio de las clínicas de lactancia materna y desarrollo, así como de los servicios de neonatología y maternidad que existen en todo el territorio nacional, se espera asuman el papel de promotores de la herramienta. Asimismo, al encontrarse la herramienta en línea existen convenios con otras universidades centroamericanas para capacitarlas en su uso y promover la atención temprana en toda la región. 
Como parte del proceso de construcción, se requiere desarrollar primeramente una revisión sistemática de investigaciones latinoamericanas sobre la aplicación de escalas de desarrollo y la atención temprana como formas de prevenir a futuro una condición de discapacidad. Para ello se parte de dos preguntas básicas: ¿qué resultados se han obtenido en Latinoamérica y España con la aplicación de algunas de las escalas de desarrollo como medio de evaluación de la primera infancia expuesta a factores de riesgo biológico neonatal?, y ¿qué efectos tiene la atención temprana en la niñez que presenta riesgo biológico neonatal para la prevención de la discapacidad?

Se presentan en este artículo los resultados de esta revisión, destacando los elementos y las experiencias que se consideran más vinculantes con el tema de la estimulación y la atención temprana como estrategia de prevención de la discapacidad. Para ello, se analizan doce investigaciones de diferentes países latinoamericanos como Chile, Perú y Argentina; así como de España. Posteriormente, se propone, a partir de la discusión de los resultados, las conclusiones que orienten el trabajo en la siguiente etapa de la investigación.

\section{ESTADO DE LA CUESTIÓN}

En Costa Rica se han desarrollado diferentes investigaciones relacionadas con este estudio. Sin embargo, no se encontró ninguna que haga referencia a los efectos de la atención temprana como forma de prevención de la discapacidad en la niñez que presenta riesgo biológico neonatal. Se presentan algunos de los trabajos más recientes realizados en el país sobre discapacidad, estimulación temprana y prematuridad.

En el año 2006, Campos, Cordero, Rojas y Vargas desarrollan la investigación "Ludoterapia y estimulación temprana: atención del niño y la niña hospitalizado (a) y su familia". El objetivo del trabajo fue incorporar la ludoterapia y la estimulación temprana en la atención de enfermería de la niñez hospitalizada en los servicios de pediatría, neonatología y maternidad del Hospital San Rafael de Alajuela, para lo cual se capacitó a un grupo de 12 miembros del personal de enfermería (supervisora, profesionales y personal auxiliar).

Entre las conclusiones de la investigación citada cabe mencionar que la falta de conocimiento sobre la ludoterapia impide desarrollar estrategias para implementarlas en el ambiente hospitalario. Se comprobó que cuando el personal de enfermería realiza la intervención con ayuda de la ludoterapia, la tarea se aliviana y resulta en beneficios para la salud mental, logrando canalizar la ansiedad y el estrés. Se considera factible incorporar la ludoterapia en el proceso de atención de la enfermería. Por último, se recomienda la distribución e implementación del manual elaborado en los servicios infantiles de los diferentes hospitales del país (Campos, Cordero, Rojas y Vargas, 2006)

En el año 2011, se publicó la "Guía para la elaboración de planes de estimulación para la promoción del desarrollo infantil de niños y niñas de 1 a 6 años y 11 meses" elaborada por un grupo de profesionales en Educación Preescolar, Psicología y Terapia de Lenguaje de la Dirección Regional de CEN-CINAI Central Sur. Se utilizaron, como punto de partida, algunos instrumentos que ya se aplicaban en el país con anterioridad: "Test y escala del desarrollo para niños y niñas de 2 a menos de 7 años", la "Guía curricular para el desarrollo integral del niño menor de seis años" y la "Escala de evaluación del niño de 0 a 6 años".

Durante el año 2009, se llevaron a cabo 15 sesiones de trabajo destinadas a la revisión bibliográfica, luego de las cuales se acordó que la guía debía incluir la misma clasificación de las áreas del desarrollo de la Escala de Evaluación del Desarrollo (EDIN) utilizada en los CEN-CINAI: motora gruesa, motora fina, cognoscitiva, lenguaje, socio afectiva y hábitos de salud. Finalizada la etapa de confección de la Guía, se procedió a su aplicación a una muestra de la población infantil de los ocho CEN-CINAI participantes; posteriormente se realizó una sesión de conversatorio.

Entre las conclusiones obtenidas se señaló que las actividades y conductas propuestas resultan de fácil comprensión para los participantes, así como para el personal, las instrucciones, el uso y el tipo de materiales. Por estas razones, la guía se dio por validada y se procedió a su publicación y difusión.

En ese mismo año, Baxter, Madriz y Mora publicaron el artículo "Prematuridad y estimulación temprana: ¿un binomio determinante para la prevención de la discapacidad? En él se realiza una revisión sistemática de investigaciones relacionadas con la aplicación de la estimulación temprana como estrategia para la prevención de la discapacidad en niñas y niños prematuros tanto en centros hospitalarios públicos como 
en el sector educativo. Entre las conclusiones destaca que la prevención de la discapacidad por medio de la estimulación temprana es esencial y que requiere de la capacitación y recursos para los profesionales encargados y los padres de familia. Asimismo se subraya que la estimulación temprana iniciada en etapas tempranas logra un mayor impacto debido a la plasticidad del sistema nervioso central. Por último, se establece la necesidad de desarrollar mayores investigaciones sobre el tema ya que en el contexto costarricense se dispone de pocos aportes.

En el año 2013 se conocieron los resultados de la investigación titulada "Estudio comparativo acerca de la implementación de programas de estimulación temprana para niños prematuros atendidos en el Estancia Infantil No 2 de la ciudad de Puebla (México) y el CINAI de la ciudad de Goicoechea (Costa Rica): una propuesta de atención temprana para la prevención de la discapacidad" desarrollada entre los años 2011 y 2013. El equipo de investigadores estuvo conformado por Jorge Baxter, Linda Madriz, Lady Meléndez, Lorena Mora, Rosa María Hidalgo y Viviana Solano.

Entre los resultados obtenidos se debe mencionar los siguientes que la atención temprana debe ser considerada como una obligación, no una opción, para la prevención o disminución de una posible alteración del desarrollo. Se reconoce la relación entre prematuridad, riesgo biológico y discapacidad en el área de salud, pero no está claro el protocolo que se debe llevar a cabo para la atención temprana de la niñez con una condición de prematuridad. Por otra parte, se encontró que no existe coordinación entre los sectores de salud y educación referente a este tema, pero sí se cuenta con una visión clara de lo que se requiere en este proceso. Es así como se plantea la importancia de establecer equipos de trabajo con miembros de ambas áreas para la atención de esta población, que además, deberá orientar y capacitar a las familias para involucrarlas en el proceso. Por último, se considera que un protocolo de atención temprana va a facilitar los procesos de inclusión al sistema educativo formal de la niñez (Baxter et al., 2013).

\section{MARCO TEÓRICO}

Se presentan en este apartado algunos de los conceptos fundamentales que favorecen la comprensión de la temática tratada.

\section{Desarrollo Infantil}

El desarrollo se concibe como el "proceso de cambio mediante el cual un niño(a) aprende a lograr mayor complejidad en sus movimientos, pensamientos, emociones y relaciones con otros" (León et al., 2004, p.16), Fundamentalmente comprende cuatro áreas: motora, cognitiva, psicoemocional y social.

Una de las principales características es su carácter dinámico y complejo ya que la interacción de los aspectos biológicos, sociales y psicológicos origina las habilidades y destrezas que permiten al niño relacionarse con el medio en forma progresiva, donde cada etapa sienta las bases de la siguiente y así sucesivamente. La gestación y los primeros años de vida constituyen un período crítico en el desarrollo ya que el cerebro experimenta un crecimiento rápido.

\section{Plasticidad cerebral}

El cerebro está formado básicamente por células nerviosas llamadas neuronas y por las fibras de conexión entre ellas. Las investigaciones recientes han demostrado que el cerebro no es estático, por el contrario es muy plástico, es decir "se va adaptando durante toda la vida a las condiciones y características del entorno... son las experiencias de cada ser humano lo que va conformando su cerebro en algo específico y propio" (Spitzer, 2005, p.94).

Los investigadores han acuñado el término neuroplasticidad o plasticidad cerebral para hacer referencia, entonces, a la capacidad de las células nerviosas para regenerarse anatómica y funcionalmente y producir nuevas conexiones neuronales. Durante la gestación y los primeros años, en el cerebro se produce una mielinización elevada, un incremento masivo y rápido de las neuronas y de las conexiones sinápticas que posibilita la adquisición, la asimilación, el aprendizaje así como la recuperación de la información de forma acelerada, sin gran esfuerzo (Fernández, 2008 y López, 2012). Así, "el cerebro puede ser modificado y modulado por las influencias ambientales, y sus propiedades plásticas permitirán compensar o corregir, en grado variable, la pérdida ocasionada por una lesión" (Malea, García, Corbí, Alemany, Fernández y Castelló, 2012, p.410). 
Para Fernández (2008), la plasticidad cerebral permite cambios en las estructuras que posibilitan la habilitación y la rehabilitación de todas las funciones, tanto las que evolucionan normalmente como aquellas defectuosas que requieran ser compensadas.

\section{Prematuridad}

La prematuridad hace referencia al nacimiento que ocurre antes de las treinta y siete semanas de gestación. El concepto de "pretérmino" por su parte, no implica la valoración de la madurez como lo hace el término "prematuro". La dificultad para reconocer la edad gestacional ha ocasionado que se utilice el peso como parámetro de clasificación, por lo que se ha acuñado el término "bajo peso al nacer" para hacer referencia a los recién nacidos con un peso inferior a los 2500 gramos. "Estos niños(as) tienen mayor riesgo a enfermar, morir o tener secuelas en su crecimiento y desarrollo" (León et al., 2004, p.62).

De acuerdo con lo establecido por Moya, Mata, Chaves y Ulate (2012) en el "Manual implementación clínicas de lactancia materna y desarrollo. Escenario hospitalario", en el año 2010, del total de 65881 nacimientos ocurridos en los hospitales de la Caja Costarricense del Seguro Social, 7,4\% (4855) tuvo un peso menor a los 2500 gramos en el momento de su nacimiento; en ese mismo período, el número de nacimientos prematuros fue de 12 222. La mayoría de los cuales presentan riesgos en su crecimiento y desarrollo, lo cual puede implicar la presencia de secuelas permanentes.

\section{Riesgo biológico neonatal}

Se considera que la niñez presenta riesgo biológico cuando durante el período pre, peri, postnatal o durante el desarrollo temprano ha estado expuesta a situaciones que podrían alterar su proceso madurativo. Entre los factores de riesgo biológico se pueden mencionar: la prematuridad, el bajo peso, la anoxia al nacer, bajas puntuaciones en la prueba Apgar y sepsis neonatal. Ello ocasiona un aumento en la probabilidad de que la persona presente alguna deficiencia o alteración posteriormente.

\section{Atención temprana}

El concepto de atención temprana se define como:

El conjunto de intervenciones dirigidas a la población infantil de 0-6 años, a la familia y al entorno, que tienen por objetivo dar respuesta lo más pronto posible a las necesidades transitorias o permanentes que presentan los niños con trastornos en su desarrollo o que tienen el riesgo de padecerlos... (GAT, 2005, p. 12)

Actualmente, se reconocen tres niveles relacionados con los procesos de estimulación en el desarrollo infantil. El primero corresponde a la estimulación temprana que se dirige a la población con o sin riesgo biológico. El segundo nivel, la estimulación orientada a la niñez que presenta una condición de riesgo biológico neonatal. Por último, el tercer nivel, dirigida a quienes presentan una condición de discapacidad. Además, se reconoce que las familias pueden desarrollar prácticas de estimulación y atención temprana, con la debida orientación. En este sentido, Quispe (2012) señala que "existe relación estadísticamente significativa entre el nivel de conocimiento de los padres sobre estimulación temprana con el desarrollo psicomotor de los niños" (p.73).

Estos procesos de estimulación, atención e intervención temprana cobran aún mayor importancia a partir de los estudios sobre la neuroplasticidad. En los primeros años de vida, las conexiones neuronales son estimuladas por factores ambientales, con lo cual se favorece el aprendizaje de determinadas habilidades, además de reducir los efectos de una deficiencia. En este período hay una mayor sensibilidad a ciertos estímulos que permiten la adquisición o estabilización de determinados tipos de conducta; así como acciones de habilitación y rehabilitación de las funciones. "La epigenética permite añadir una gran dosis de indeterminismo a la genética y refuerza la importancia del ambiente" (Méndez, 2010, párr.8). Es así como cada vez más se reconoce que el entorno modifica el papel de la genética.

\section{METODOLOGÍA Y MATERIALES}

De acuerdo con Ferreira, Urrútia y Alonso-Coello (2011, párr. 1) la revisión sistemática o metaanálisis es "una herramienta esencial para sintetizar la 
información científica disponible, incrementar la validez de las conclusiones de estudios individuales e identificar áreas de incertidumbre donde sea necesario realizar investigación".

En el presente trabajo se revisaron sistemáticamente algunas experiencias en Latinoamérica y España referentes a la aplicación de las escalas de desarrollo infantil y de la atención temprana como estrategia para la prevención de la discapacidad.

Dentro de los objetivos principales se halla el determinar los resultados obtenidos en la aplicación de escalas de desarrollo en la primera infancia en Latinoamericana y España que se constituyan en insumos para la construcción de la herramienta web dirigida a las familias y a los cuidadores de la niñez que presenta riesgo biológico neonatal. De igual manera, el identificar la forma en que la atención temprana se constituye en una estrategia para la prevención de la discapacidad en las investigaciones consultadas.

A partir de estos objetivos se propusieron las siguientes preguntas orientativas: ¿qué resultados se han obtenido en Latinoamérica y España con la aplicación de algunas de las escalas de desarrollo como medio de evaluación de la primera infancia expuesta a factores de riesgo biológico neonatal? y ¿qué efectos presenta la atención temprana en la niñez que presenta riesgo biológico neonatal para la prevención de la discapacidad?

Se establecieron como fuentes para recabar la información las bases de datos en línea EBSCO y ProQuest, las bibliotecas del Centro Nacional de Recursos para la Educación Inclusiva, de la Universidad de Costa Rica y de la Universidad Estatal a Distancia, la consulta y el préstamo interbibliotecario así como la consulta de documentos en línea. Como método, se utilizó la búsqueda aleatoria a partir de filtros con palabras clave afines a los objetivos a nivel de Latinoamérica y de España.

Los documentos se seleccionaron a partir de tres criterios: temática expuesta afín a la investigación; actualidad, los documentos debían ser elaborados en el período 2005-2015 y de origen latinoamericano o español.

Es así como, luego de identificar potenciales estudios, se procedió a su análisis crítico. Para ello se compararon las investigaciones utilizando matrices que incluían los tres criterios establecidos y contra teoría. Primero, en forma independiente (por parte de cada investigadora), para llegar luego a un consenso con el fin de aumentar la confiabilidad y la seguridad del proceso.

Se eligieron finalmente doce investigaciones de diferentes países, entre los que cabe mencionar a Chile, Perú, Argentina y España. Por las características de esta revisión sistemática, se considera que los artículos seleccionados cumplen con los criterios establecidos, son pertinentes por su carácter académico o científico y además resultan válidos para efectos del presente trabajo. En la fase de extracción y síntesis de los resultados de los estudios se definió de manera consensuada entre las autoras la información por recopilar: escala de desarrollo utilizada, población, método empleado, resultados obtenidos, así como conclusiones y recomendaciones sobre la aplicación de la escala y los efectos de la estimulación y atención temprana en la primera infancia. Al final del artículo, se presentan los resultados, la discusión y las conclusiones de las autoras luego de la revisión sistemática de los estudios.

Como se indicó anteriormente, el presente trabajo surge como parte de la primera fase de la investigación orientada a la construcción de una herramienta tecnológica para la atención temprana. A nivel metodológico, se desarrolló desde un enfoque mixto ya que recolecta, analiza y vincula datos cuantitativos y cualitativos en un mismo estudio para dar respuesta a un problema determinado (Hernández, 2008). Se estructuró en cuatro fases: una primera fase en la que se recolecta la información necesaria para la construcción de la herramienta tecnológica de valoración y atención temprana. La segunda, propone la construcción, implementación y validación de los instrumentos. La tercera fase implica el pilotaje de los instrumentos de la herramienta como parte del proceso de validación. Por último, en la cuarta fase, se propone determinar el efecto en el desarrollo de los niños con riesgo biológico neonatal participantes, después de recibir la atención temprana empleando la herramienta de diseño longitudinal.

Tal y como afirma Hernández (2008), los diseños longitudinales "...recolectan datos a través del tiempo en puntos o períodos, para hacer inferencias respecto al cambio, sus determinantes y consecuencias" (p.216).

\section{RESULTADOS}

Las escalas de desarrollo que proporcionan datos relevantes para la atención temprana en la primera 
infancia y el efecto de este tipo de estimulación en el desarrollo de la niñez han sido objeto de estudio desde diferentes enfoques y disciplinas. Para efectos de este trabajo se llevó a cabo una revisión sistemática de algunas de las investigaciones latinoamericanas realizadas en los últimos diez años sobre estos temas.

En el año 2005 en la provincia de Córdoba (Argentina), Rodríguez, Calderón, Cabrera, Ibarra, Moya y Faas desarrollaron el "Análisis de consistencia interna de la Escala Bayley del desarrollo infantil para la ciudad de Córdoba (Primer Año de Vida)", mediante el cual se pretende demostrar la confiabilidad para, en una segunda etapa, realizar la adaptación del instrumento que permita medir el desarrollo infantil en una forma práctica y objetiva. La escala se aplicó a una muestra accidental de 150 infantes de ambos sexos, con edades entre el mes y medio y el año y quince días, nacidos a término y con una talla y perímetro cefálicos adecuados para la edad gestacional. Entre los resultados obtenidos, se plantea que la aplicación de la escala permite la evaluación global infantil, desarrolla el interés de los padres hacia la estimulación de sus hijos y "crea un espacio de reflexión acerca del desarrollo del niño, lo que abre caminos a la prevención" (Rodríguez, Calderón, Cabrera, Ibarra, Moya y Faas, 2005, p.12). Los autores citados concluyen que realizar "la detección de demoras en el desarrollo posibilita por consiguiente la intervención temprana, siendo este el camino más efectivo para que el niño pueda desarrollar al máximo sus potencialidades" (Rodríguez, Calderón, Cabrera, Ibarra, Moya y Faas, 2005, págs. 12, 13).

También en el 2005, en Medellín, Atehortúa realiza la investigación "La estimulación temprana del neonato hospitalizado en la unidad de cuidados intensivos neonatales", en la cual aplica una encuesta al personal de enfermería, y obtiene, entre otras, las siguientes conclusiones: los participantes consideran que la estimulación temprana es una intervención que coadyuva al desarrollo del neonato, pero se presenta en forma empírica. Por ello se requiere brindar la capacitación necesaria de modo que se pueda incluir en los planes de cuidado diario de la niñez. La investigadora considera que se requiere la evaluación del neonato por medio de una escala de evaluación del desarrollo y que la participación de la familia es esencial, pues es la que debe continuar con la estimulación temprana al egreso de la unidad de cuidados intensivos. Los familiares deben también capacitarse, deseablemente durante la estadía hospitalaria. Por último, se recomienda la promoción de investigaciones sobre la estimulación temprana desde el campo de la Enfermería, ya que no existen muchos estudios al respecto.

En el año 2006, Bolaños, Golombek, Márquez, de la Riva, Sánchez, Gutiérrez y Elorza desarrollan en México el estudio "Validez de correlación del perfil de conductas de desarrollo con la Escala de Desarrollo Infantil Bayley II", con el objetivo de determinar si ambos instrumentos miden constructos similares sin que se presenten diferencias significativas en el análisis de medias. Las escalas se aplicaron a 40 participantes de ambos sexos con edades entre 1 a 42 meses de edad. Los resultados señalan que ambas pruebas detectan a la niñez que presenta problemas en las subescalas establecidas. Se comprobó que las dos miden constructos similares y que existe correlación entre las áreas del perfil de conductas de desarrollo. El estudio amplía la validez del PCD-R como instrumento diagnóstico de retrasos y alteraciones en el desarrollo.

Por su parte, siempre en el año 2006, Sánchez realiza la investigación "La eficacia de los programas de atención temprana en niños de riesgo biológico. Estudio sobre los efectos de un programa de atención temprana en niños prematuros en su primer año de vida" como tesis doctoral en la Universidad de Murcia, España. Participaron 116 infantes divididos en un grupo de nacidos a término y otro de prematuros o bajo peso. Entre las conclusiones cabe mencionar que los nacimientos prematuros suelen presentar un riesgo de discapacidad en función del grado de alteración biológica por lo que es deseable que sean evaluados, seguidos y tratados lo antes posible, incluso desde el alta hospitalaria y durante toda la primera infancia. Sin embargo, de acuerdo con los resultados obtenidos, la condición biológica no siempre predice la evolución, ya que influyen factores ambientales de crianza y estimulación que pueden incidir en su progreso.

El autor en mención considera importante intervenir sobre el estrés materno de manera temprana ya que se comprobó que influye de manera indirecta en el desarrollo del niño. También señala que existe un enlentecimiento en el desarrollo de los niños prematuros que confirma la posibilidad de que existan discontinuidades en éste; en relación con la eficacia de los programas de atención temprana, se concluye que los tres primeros años de vida es el plazo requerido para que los niños prematuros se acerquen a la norma. 
Ramos, Cruz, Pérez, Salvatierra, Robles, Koletzko, Decsi y Campoy desarrollan en el año 2008 la investigación "Predicción del desarrollo mental a los 20 meses de edad por medio de la evaluación del desarrollo psicomotor a los seis meses de vida en niños sanos". Los resultados mostraron que el desarrollo psicomotor fue mejor predictor del desarrollo mental a los seis meses que el propio desarrollo mental a esa edad.

En el año 2009 se pone en práctica el Programa Regional de Indicadores de Desarrollo Infantil (PRIDI), como una iniciativa para recopilar datos sobre el desarrollo infantil en Costa Rica, Nicaragua, Paraguay, Brasil y Perú. La información permite reconocer las brechas en el desarrollo infantil entre diferentes poblaciones y zonas, de modo que posteriormente se implementen planes para su reducción. Por otra parte, se pretende obtener datos comparables a nivel regional, constituyéndose en el primer estudio internacional de su tipo.

El programa consta de tres fases en las que los instrumentos fueron aplicados a diferentes tipos de muestras. Al finalizar las dos primeras fases se realizaron ajustes en las encuestas y las escalas. Estas últimas fueron denominadas formalmente como Escalas Engle de Desarrollo Infantil. La última fase del programa, la recopilación de los datos, se encuentra en proceso y en ella se espera la recolección de datos de dos mil infantes de cada país para su posterior análisis.

En ese mismo año, Schonhaut, Salinas, Armijo, Schönstedt, Álvarez y Manríquez llevan a cabo la investigación "Validación de un cuestionario autoadministrado para la evaluación del desarrollo psicomotor". El objetivo del estudio es evaluar las propiedades psicométricas del ASQ (Ages and Stages Questionnaries), cuestionario de auto-reporte de la familia para luego compararlo con la Escala de Bayley III de Desarrollo Infantil (BSID), la cual fue aplicada por un experto, ciego ante los resultados del ASQ.

El estudio transversal analítico se realiza con 119 infantes en una clínica privada en Santiago de Chile. Se comprobó una adecuada correlación entre el ASQ y el BSID, se concluye además que el primero de ellos podría ser utilizado como un método de proyección ("screening") del desarrollo psicomotor en la consulta pediátrica.

En otro estudio realizado por Reyna y Brussino (2009) denominado "Propiedades psicométricas de la escala de comportamiento preescolar y jardín infantil en una muestra de niños argentinos de 3 a 7 años", se aplica además, un cuestionario sociodemográfico a la familia. Entre los resultados obtenidos se ratifica que la escala se considera adecuada para evaluar el comportamiento social en el rango de edad indicado. Destaca la importancia de contar con el informe de la familia ya que proporciona datos sobre el comportamiento en otro ambiente.

En el año 2010 Quezada, Soto, Escobar y López desarrollan la investigación "Confiabilidad interevaluador de la Escala Motora Infantil de Alberta (AIMS) a niños de término y pretérmino de la provincia de Talca, Chile" con 115 participantes de entre 10 y 16 meses. Se realiza un diseño de corte transversal en el que se filma su conducta motora gruesa, evaluada posteriormente por dos observadores entrenados en la escala. Los investigadores concluyen que la AIMS tiene un excelente grado de confiabilidad interobservador. Además, ante la carencia de escalas motoras validadas en Chile, se debe continuar investigando este instrumento de evaluación para dar soporte adicional a su aplicación en la práctica.

De igual forma, Pollitt y Caycho (2010) realizan el estudio "Desarrollo motor como indicador del desarrollo infantil durante los primeros dos años de vida" en Perú. Entre las conclusiones se plantea que en Perú no existen políticas que fomenten el desarrollo infantil y prevengan su rezago, pero es posible inferir que este no sigue el curso esperado en las zonas de pobreza extrema. Basados en la evidencia científica consideran que el desarrollo motor es el indicador más apropiado del desarrollo infantil en los dos primeros años de vida.

Por su parte, en el año 2011 Oré, Díaz y Penny desarrollaron la investigación "Impacto de una intervención con grupos de mamás y bebés en el desarrollo infantil" en una zona marginal de Lima, Perú. Se contó con 163 participantes con edades entre los ocho y once meses de edad con sus respectivas madres. Se evalúa su desarrollo antes y después de la intervención con la escala BSID-II. Se desarrollan sesiones grupales donde cada madre y su hijo llevan a cabo diferentes actividades de estimulación. Los resultados apuntan a que la intervención tiene un impacto general positivo en el desarrollo de los infantes, se halla un efecto significativo en los factores de regulación emocional y calidad motora asociados con el impacto producido en la interacción madre-hijo. Otra conclusión a la que se llega es que se carece de instrumentos que evalúen el desarrollo infantil en forma válida y confiable. 
En ese mismo año, Noheda, Mateos, Valle, Moro, Portellano y Martínez en la investigación "Rendimiento neuropsicológico en niños pretérmino con muy bajo peso en el nacimiento" evalúan las características neuropsicológicas en dos grupos: uno de control y otro de bajo peso. En total participaron 73 niños con edades promedio entre los 31 y 32 meses a los que se les aplicó una batería de pruebas de valoración del desarrollo.

El grupo control obtuvo mejores resultados en casi todas las funciones, aunque no todas fueron estadísticamente significativas. Se concluye que el bajo peso tiene un efecto negativo en el desarrollo infantil y que la condición de prematuridad provoca un riesgo mayor de presentar trastornos neuropsicológicos y a futuro desarrollar otras patologías.

Por otra parte, los autores plantean que "la preocupación por la recuperación de estas poblaciones de riesgo biológico es escasa y limitada sólo a determinados profesionales. Los padres y profesores no conocen el alcance y la problemática de estos niños" (Noheda, Mateos, Valle, Moro, Portellano y Martínez, 2011, p.144). Por último, proponen que la estimulación temprana disminuye el riesgo de fracaso escolar y dificultades de aprendizaje debido a la plasticidad cerebral en los primeros años de vida.

\section{DISCUSIÓN}

Con el propósito de discutir los resultados se retoma el análisis realizado y se propone una triangulación con la teoría. Por las características del trabajo (revisión sistemática), se procede a señalar los aportes de los estudios presentados que se consideran de relevancia para los objetivos de la investigación.

El meta-análisis elaborado permite establecer tres ejes temáticos: la aplicación de escalas de desarrollo a poblaciones menores de seis años, la estimulación y la atención temprana dirigida a la niñez con riesgo biológico y la participación de la familia en este proceso.

En relación con la aplicación de escalas de desarrollo, las investigaciones se orientan principalmente a determinar su validez y confiabilidad; en algunos de los casos como paso previo para hacer una adaptación a la realidad del país. Se hace evidente la necesidad de contar con datos que proporcionen información sobre el desarrollo infantil, pero se enfatiza en que no se dispone de estudios suficientes al respecto. Algunos autores plantean la valoración del desarrollo como paso previo para la implementación de planes de estimulación en las áreas en que se presente un bajo desempeño.

En el proceso de búsqueda y revisión bibliográfica, únicamente se encontraron dos estudios referidos a la estimulación temprana de la niñez que presenta riesgo biológico neonatal, pero no mencionan directamente el efecto de ésta como estrategia de prevención de la discapacidad. Sin embargo, Nohela et al. (2011) plantean que el bajo peso y la prematuridad pueden tener efectos negativos en el desarrollo infantil y en la aparición de secuelas. Sánchez (2006) por su parte profundiza al plantear que la niñez que nace prematura presenta riesgo de sufrir una discapacidad, aunque contempla los factores ambientales como forma de incidir en el progreso. Considera que el infante prematuro puede llegar a acercarse al desarrollo esperado cerca del tercer año de vida si ha recibido estimulación temprana desde bebé. Estos resultados coinciden con Moreno (2011, p.78) al señalar que luego de un programa comunitario de atención temprana se "alcanzó mejoría en el neurodesarrollo en la mayoría de los casos, tanto en los pacientes como en los niños con factores de riesgo pero sin retraso del neurodesarrollo, sobre todo en la esfera motora".

Los estudios enfatizan la importancia de que la familia y los cuidadores se incorporen en la estimulación y atención temprana de la niñez desde que se encuentra en el hospital. Por otra parte, una de las investigaciones comenta el efecto positivo que posee la interacción madre-hijo en el desarrollo infantil.

También se desprende de la revisión la necesidad de capacitar a los profesionales, familias y cuidadores en el tema de la estimulación y atención temprana. En este sentido Osorio, Torres-Sánchez, Hernández, LópezCarrillo y Schnaas (2010, p.21) consideran que es necesario "establecer programas de educación a padres que fomenten la estimulación de los niños en casa para favorecer su desarrollo integral". Este criterio es compartido por Chávez (2014, p.88) al señalar que "la implementación de un programa de capacitación en estimulación temprana contribuye a que los padres y/o encargados brinden una atención adecuada a sus hijos/as y que presenten un mejor desarrollo de sus capacidades".

Excepto el Programa Regional de Indicadores de Desarrollo Infantil (PRIDI) que contempla a Costa Rica y 
a Nicaragua como parte de la investigación, las demás investigaciones se realizaron en países de Suramérica, México y España. No se hallaron estudios sobre los temas tratados desarrollados a nivel centroamericano.

\section{CONCLUSIONES}

De la revisión sistemática efectuada, se desprenden las conclusiones que se exponen seguidamente en este apartado.

En los diferentes países de Latinoamérica y España los estudios que se realizan sobre escalas de desarroIlo infantil se circunscriben más a establecer la validación y la confiabilidad que permitan eventualmente la adaptación del instrumento al contexto. Sin embargo, no trascienden en el uso de los instrumentos como una herramienta de empoderamiento de las familias y cuidadores para promover la estimulación y atención temprana de la niñez ante una condición de riesgo biológico.

En algunos casos, se han elaborado instrumentos de evaluación del desarrollo infantil, pero las investigaciones de igual manera se orientan a correlacionarlos con otros reconocidos internacionalmente para determinar su validez.

Se subraya en los estudios la importancia de evaluar el desarrollo infantil, en particular cuando se presenta riesgo biológico neonatal. Los resultados obtenidos se constituyen en el insumo para la elaboración de planes de atención dirigidos a las áreas deficitarias.

Por otra parte, la revisión sistemática señala la importancia que se le concede a la estimulación y atención temprana por los beneficios que puede generar a la población infantil. No obstante, en los estudios no se contempla el abordaje educativo, se requiere desarrollar procesos sistemáticos de capacitación dirigidos a los profesionales encargados del cuido y atención de los infantes en los servicios de salud..

Resalta también en la literatura analizada el papel que se concede a la participación de la familia en la estimulación temprana de los niños. Reviste interés e importancia su formación en el tema con el fin de que presenten mejores condiciones para trabajar con sus hijos.
No se encontraron investigaciones en el contexto de Latinoamérica o España que aborden directamente el tema de la estimulación temprana como estrategia de prevención de la discapacidad, ni estudios longitudinales que permitan determinar a largo plazo la evolución de la niñez que recibe este tipo de atención así como la minimización o eliminación de las secuelas asociadas a los factores de riesgo biológico neonatal. En este contexto, cobra importancia la investigación que se desarrolla en Costa Rica, ya que por una parte se va a diseñar la herramienta web, pero también el estudio longitudinal permite contar con datos científicos que respalden la aplicación de la estimulación temprana como forma de prevenir una condición de discapacidad.

Resulta preocupante el hecho de que no se encontraran investigaciones centroamericanas sobre los temas en cuestión, lo cual es una llamada de alerta para promover procesos investigativos a nivel nacional y deseablemente en el resto de los países de la región. La investigación costarricense se constituye en innovadora y pionera tanto en el área como en el país; además ofrece datos contextualizados que pueden servir de base para otros estudios regionales.

Como conclusión general, cobra importancia el hecho de que la investigación costarricense que la originó se vaya a concretar con el fin de construir e implementar la herramienta virtual de valoración y estimulación temprana, así como la realización posterior del estudio longitudinal que permita determinar su efecto en la prevención de la discapacidad.

\section{REFERENCIAS}

Atehortúa, S. (2005). La Estimulación Temprana del neonato hospitalizado en la Unidad de Cuidados Intensivos Neonatales. (Monografía para optar al título de Especialista en cuidado al niño en estado crítico de salud Universidad de Antioquía). Recuperado de http:// tesis.udea.edu.co/dspace/bitstream/10495/150/1/ EstimulacionNeonatoHospitalizado.pdf

Baxter, J., Madriz, L., Meléndez, L. Mora, L., Hidalgo, R.M., Solano, V. (2011). Estudio Comparativo acerca de la implementación de programas de Estimulación Temprana para niños prematuros atendidos en el Estancia Infantil No 2 de la ciudad de Puebla (México) y el CINAI de la ciudad de Goicoechea (Costa Rica): una propuesta de Atención Temprana para la prevención de la discapacidad. Universidad Estatal a Distancia. Documento inédito. 
Baxter, J., Madriz, L., Meléndez, L., Mora, L., Hidalgo, R.M., Solano, V. (2013). Análisis de resultados. Estudio Comparativo acerca de la implementación de programas de Estimulación Temprana para niños prematuros atendidos en el Estancia Infantil \#2 de la ciudad de Puebla (México) y el CINAI de la ciudad de Goicoechea (Costa Rica): una propuesta de Atención Temprana para la prevención de la discapacidad. Documento inédito

Baxter, J., Madriz, L., Mora, L. (2011). Prematuridad y estimulación temprana: ¿un binomio determinante para la prevención de la discapacidad?. Revista Innovaciones Educativas Año XIII (18) pp. 11-21. Recuperado de http:// www.uned.ac.cr/ece/images/revista/numero\%2018\%20 2012/01-Baxter-Prematuridad.pdf

Bolaños, C., Golombek, L., Márquez, A., de la Riva, M., Sánchez, C.E., Gutiérrez, O. y Pereztejada, H. (2006). Validez de correlación del Perfil de Conductas de Desarrollo con la Escala de Desarrollo Infantil Bayley II. Acta Pediátrica de México, 27(4), pp 190-199. Recuperado de http:// web.ebscohost.com/ehost/detail?vid=6\&sid=19d01ea9b042-4d8f-8b305911dc126c85\%40sessionmgr111\&hid= 120\&bdata $=$ Jmxhbmc9ZXMmc2I0ZT1laG9zdC1saXZI\#d $b=\mid$ th\&AN $=22571354$

Campos, C., Cordero, S., Rojas, G., Vargas, M. (2005). Ludoterapia y estimulación temprana: atención del niño y la niña hospitalizado (a) y su familia. Revista de Enfermería ISSN 1409-1992/2005/26(1) pp. 5-12. Recuperado de http:// revista.enfermeria.cr/sites/default/files/Ludoterapia\%20 y\%20Estimulaci\%C3\%B3n\%20Temprana.pdf

Chávez, A. (2014). Elaboración de un programa de un programa de estimulación temprana en las áreas cognoscitivas, socioafectivas y psicomotriz dirigido a padres y/o encargados de niños de 18 a 36 meses, que asisten al centro de desarrollo infantil San Sebastián Mártir, de Ayutuxtepeque, San Salvador. Recuperado de http:// ri.ues.edu.sv/5559/1/Elaboraci\%C3\%B3n\%20de\%20 un $\% 20$ programa\%20de\%20capacitaci\%C3\%B3n\%20 en\%20estimulaci\%C3\%B3n\%20temprana\%20en\%20 las $\% 20 \%$ C3\%A1reas\%20cognoscitivas,\%20socioafectivas\%20y\%20psicomotriz\%20dirigido\%20a\%20padres $\% 20$ yo\%20ncargados $\% 20$ de $\% 20$ ni\%C3\%B1os $\% 20$ de\%2018\%20a\%2036\%20meses,\%20que\%20asisten\%20al\%20Centro\%20de\%20D.pdf

Federación Estatal de Asociaciones de Profesionales de Atención Temprana (GAT) (2005). Libro Blanco de la Atención Temprana. Recuperado de www.fcsd.org/fichero69992_69992.pdf

Fernández, Y. (2008). Atención Temprana: prevención de las Necesidades Educativas

Especiales (NEE). Ef deportes revista digital, 12(118). Recuperado de http://www.efdeportes.com/efd118 atencion-temprana-prevencion-de-las-necesidadeseducativas-especiales.htm

Ferreira,I.,Urrútia, G.y Alonso-Coello,P.(2011). Revisiones sistemáticas y metaanálisis: bases conceptuales e interpretación. Revista Española de Cardiología, 64(8), pp.688-696. Recuperado de http://www.revespcardiol.org/es/revisiones-sistematicas-metaanalisis-bases-conceptuales/ articulo/90024424/

Katz, L. (2005). Perspectivas Educativas en la Primera Infancia. Chile: LOM Ediciones Ltda.

León, M. et al. (2004). Crecimiento y desarrollo en la niñez y abordaje de los principales trastornos. Módulo VII. EDNASSS-CCSS. Recuperado de http://www.cendeisss. sa.cr/posgrados/modulos/Modulo7/Modulo_7.pdf

Malea, I., García, R., Corbí, P., Alemany, C., Fernández, C., y Castelló, M. L. (2012). Neurología y Síndrome de Down. Desarrollo y Atención Temprana. Revista Española de Pediatría, 68(6), pp 409-414. Recuperado de http://www. sindromedown.net/adjuntos/cPublicaciones/122L_revista.pdf

Méndez, J. (2010). Las jirafas de Lamarck, los gemelos, el cáncer y la guerra en Holanda. Recuperado de http://blogs. heraldo.es/ciencia/?p=1288

Moreno, R., (2011). Eficacia de un programa comunitario de atención temprana del neurodesarrollo en el municipio Habana Vieja. 1998-2008. Recuperado de http://tesis. repo.sld.cu/519/1/MorenoMora.pdf

Moya, R., Mata, A., Chaves, S., Ulate, F. (2012). Manual Implementación Clínicas de Lactancia Materna y Desarrollo Escenario Hospitalario. Recuperado de http://www.ministeriodesalud.go.cr/gestores_en_salud/lactancia/CNLM_manual_lactac_materna_y_desarrollo_2012.pdf

Navarro, G. et al. (2011). Guía para la elaboración de planes de estimulación para la promoción del desarrollo infantil de niños y niñas de 1 a 6 años y 11 meses. Ministerio de Salud y Dirección Nacional de CEN CINAI. Recuperado de http://www.unicef.org/costarica/docs/cr_pub_Guia_ elaboracion_de_planes_estimulacion_promocion_desarrollo_infantil.pdf

Noheda Cifuentes, S. S., Mateos Mateos, R. R., Valle Trapero, M. M., Moro Serrano, M. M., Portellano Pérez, J. A., \& Martínez Arias, R. R. (2012). Rendimiento neuropsicológico en niños pretérmino con muy bajo peso en el nacimiento. (Spanish). Acta Pediátrica Española, 70(4), pp. 141-146. Recuperado de http://web.ebscohost. com/ehost/detail?vid=6\&sid=19d01ea9-b042-4d8f8b30-5911dc126c85\%40sessionmgr111\&hid=120\&bda ta=Jmxhbmc9ZXMmc2l0ZT1laG9zdC1saXZl\#db=|th\& $\mathrm{AN}=82313204$ 
Oré, B., Díaz, J. y Penny, M. (2011). Impacto de una intervención con grupos de mamás y bebés en el desarrollo infantil. (Spanish). Psicología (02549247), 29(1), pp. 3766. Recuperado de http://web.ebscohost.com/ehost/ detail?vid=6\&sid=19d01ea9-b042-4d8f-8b30-5911dc12 $6 c 85 \% 40$ sessionmgr111\&hid=120\&bdata $=$ Jmxhbmc9ZX Mmc2l0ZT1laG9zdC1saXZl\#db=a9h\&AN=64466743

Poblano, A. (2003). Detección y estimulación tempranas del niño con daño neurológico. México D.F.: Editores de Textos Mexicanos S.A

Pollit, E., Caycho, T. (2010). Desarrollo motor como indicador del desarrollo infantil durante los primeros dos años de vida. Revista de Psicología (0254-9247) 28(2), 2010 pp. 385-413 Recuperado de http://web.ebscohost. com/ehost/detail?vid=6\&sid=19d01ea9-b042-4d8f8b30-5911dc126c85\%40sessionmgr111\&hid=120\&bda ta=Jmxhbmc9ZXMmc2l0ZT1laG9zdC1saXZl\#db=a9h\& $\mathrm{AN}=56952722$

Ramos, R., Cruz, F., Pérez, M., Salvatierra, M., Robles, C., Koletzko, B. V., \& ... Campoy, C. (2008). Predicción del desarrollo mental a los 20 meses de edad por medio de la evaluación del desarrollo psicomotor a los seis meses de vida en niños sanos. (Spanish). Salud Mental, 31(1), pp.5361. Recuperado de http://web.ebscohost.com/ehost/ detail?vid=6\&sid=19d01ea9-b042-4d8f-8b30-5911dc12 $6 c 85 \% 40$ sessionmgr111\&hid $=120 \& b d a t a=J m x h b m c 9 Z X$ Mmc2I0ZT1laG9zdC1saXZl\#db=|th\&AN=31808251

Quezada-Villalobos, L., Soto-García, I., Escobar-Cabello, M., \& López-Suárez, A. (2010). 'Confiabilidad interevaluador' de la Escala Motora Infantil de Alberta en niños de término y pretérmino de la provincia de Talca - Chile. (Spanish). Revista Ciencias De La Salud, 8(2), 2132. Recuperado de http://web.ebscohost.com/ehost/ detail?vid=6\&sid=19d01ea9-b042-4d8f-8b30-5911dc12 $6 c 85 \% 40$ sessionmgr111\&hid=120\&bdata=Jmxhbmc9ZX Mmc2lo

Quispe, H.D. (2012). Nivel de conocimiento y actitud de los padres sobre la estimulación temprana en relación con el desarrollo psicomotor del niño de 4 a 5 años de la IE Jorge Chávez TACNA-2010. Recuperado de http://tesis. unjbg.edu.pe:8080/bitstream/handle/unjbg/130/24

Quispe_Gutierrez_HD_FACS_Enfermeria_2012_ Resumen.pdf?sequence $=2$
Reyna, C. y Brussino, S. (2009). Propiedades Psicométricas de la Escala de Comportamiento Preescolar y Jardín Infantil en una Muestra de Niños Argentinos de 3 a 7 Años. (Spanish). Psykhe, 18(2), pp.127-140. Recuperado de web.ebscohost.com/ehost/detail?vid=6\&sid=19d01ea9b042-4d8f-8b30-5911dc126c85\%40sessionmgr111\&hid= 120\&bdata $=$ Jmxhbmc9ZXMmc2I0ZT1laG9zdC1saXZl\#d $\mathrm{b}=\mathrm{a} 9 \mathrm{~h} \& \mathrm{AN}=50544917$

Rodríguez, M., Calderón, L., Cabrera, L., Ibarra, N., Moya, P. y Faas, A.E. (2005) Análisis de Consistencia Interna de la Escala Bayley del Desarrollo Infantil para la Ciudad de Córdoba (Primer año de Vida). Evaluar, 2005, No.5 (octubre), pp.55-69 Laboratorio de Evaluación Psicológica y Educativa. Facultad de Psicología Universidad Nacional de Córdoba. Recuperado de http://revistas.unc.edu.ar/index.php/revaluar/article/ download/540/480\%E2\%80\%8E

Sánchez, J. (2006). La eficacia de los Programas de Atención Temprana en niños de Riesgo Biológico. Estudio sobre los efectos de un programa de Atención Temprana en niños prematuros en su primer año de vida. (Tesis doctoral Universidad de Murcia). Recuperado de http://digitum. um.es/jspui/bitstream/10201/99/1/SanchezCaravaca.pdf

Schonhaut, L., Salinas, P., Armijo, I., Schönstedt, M., Álvarez, J. y Manríquez, M. (2009). Validación de un Cuestionario Autoadministrado para la Evaluación del Desarrollo Psicomotor. Revista Chilena de Pediatría, 80(6), pp. 513519. Recuperado de http://web.ebscohost.com/ehost/ detail?vid=6\&sid=19d01ea9-b042-4d8f-8b30-5911dc12 $6 c 85 \% 40$ sessionmgr111\&hid $=120 \&$ bdata $=J m x h b m c 9 Z X$ Mmc2I0ZT1laG9zdC1saXZI\#db=|th\&AN=48384032

Spitzer, M. (2005). Aprendizaje Neurociencia y la escuela de la vida. Barcelona: Ediciones Omega

Verdisco, A., Thompson, J., Hepworth, K. (2013) Programa Regional de Indicadores de Desarrollo Infantil (PRIDI): procesos, resultados y retos encontrados hasta la fecha. Espacio para la infancia Julio 2013 Fundación Bernard van Leer, pp 32-36. Recuperado de http://earlychildhoodmagazine.org/wp-content/uploads/2013/07/6.Programa-Regional.pdf

Zuluaga, J. (2001). Neurodesarrollo y estimulación. Bogotá: Editorial Médica Internacional Ltda. 